Meta

Journal des traducteurs

Translators' Journal

\title{
La recherche documentaire en traduction technique : conditions nécessaires et suffisantes
}

\section{Christine Durieux}

Volume 35, numéro 4, décembre 1990

URI : https://id.erudit.org/iderudit/002688ar

DOI : https://doi.org/10.7202/002688ar

Aller au sommaire du numéro

Éditeur(s)

Les Presses de l'Université de Montréal

ISSN

0026-0452 (imprimé)

1492-1421 (numérique)

Découvrir la revue

Citer cet article

Durieux, C. (1990). La recherche documentaire en traduction technique :

conditions nécessaires et suffisantes. Meta, 35(4), 669-675.

https://doi.org/10.7202/002688ar d'utilisation que vous pouvez consulter en ligne. 


\section{LA RECHERCHE DOCUMENTAIRE EN TRADUCTION TECHNIQUE: CONDITIONS NÉCESSAIRES ET SUFFISANTES}

CHRISTINE DURIEUX

Université de la Sorbonne Nouvelle-Paris III, Paris, France

La plupart des auteurs qui ont écrit sur la traduction technique ont toujours mis l'accent sur le vocabulaire ou la stylistique, ou les deux. Ainsi, les difficultés de la traduction technique seraient-elles réductibles aux difficultés inhérentes à l'usage d'une langue de spécialité. Or, la démarche mise en œuvre pour effectuer la traduction d'un texte technique va bien au-delà de la recherche de termes ou de tournures, elle exige une compréhension du texte. Certes, la terminologie joue un rôle important dans la traduction d'un texte technique, mais elle n'en est qu'un aspect auxiliaire et ne saurait en être l'objet principal. Les vrais problèmes de la traduction technique ne sont pas tant d'ordre lexical que notionnel, d'où la nécessité de procéder à une recherche documentaire de nature à éclairer le traducteur sur le sujet traité.

\section{CONDITIONS NÉCESSAIRES}

La recherche documentaire n'est pas un ingrédient obligatoire de la démarche traduisante appliquée à un texte technique, mais quand elle s'impose, elle occupe alors une place prépondérante dans la phase sémasiologique et joue également un rôle clé lors de la phase onomasiologique, dans laquelle elle remplace avantageusement la recherche terminologique ponctuelle.

Tout d'abord, il importe de juger de la nécessité de procéder à une recherche documentaire. À cet égard, les critères à prendre en compte ne sont pas des critères absolus : technicité du sujet ou ancienneté du traducteur dans le métier. En effet, la nécessité de procéder à une recherche documentaire n'est pas liée au texte lui-même. On ne peut pas dire que certains sujets, plus que d'autres, justifient une telle recherche. Il n'y a pas de thème qui exige systématiquement une recherche documentaire. Par ailleurs, la recherche documentaire n'est pas non plus le lot exclusif des traducteurs débutants. Il serait faux d'imaginer que les plus anciens dans la profession peuvent naturellement s'y soustraire. Le critère à retenir est de nature toute relative: la nécessité de procéder à une recherche documentaire dépend de la relation qui existe entre le traducteur et le texte à traduire.

Prenons l'exemple d'un texte traitant des trains à lévitation magnétique. Cette technique peut paraître ardue de prime abord, mais ce n'est pas pour autant que la traduction de ce texte exige une recherche documentaire systématique. Certes, le traducteur ignorant tout de cette technique aura fort à faire pour aborder ce texte dans de bonnes conditions: sa démarche sera longue, et devra être méthodique pour être efficace. Qu'il soit traducteur débutant ou confirmé, la démarche sera la même. Peut-être un traducteur expérimenté, qui maîtrise déjà la méthode de recherche documentaire pour l'avoir appliquée à d'autres disciplines techniques, peut-il avancer de façon plus rapide que le traducteur débutant, mais sans pour autant brûler les étapes; simplement en les franchissant plus vite. En revanche, le traducteur — qu'il soit débutant ou confirmé — qui s'est intéressé à 
ce nouveau moyen de transport, a vu des maquettes, a suivi des émissions de télévision sur ce sujet et a lu des articles sur le fonctionnement de ce type de train, aura éventuellement besoin d'acquérir des connaissances spécifiques complémentaires pour exécuter la traduction du texte, mais sans devoir se lancer dans la totalité de la démarche méthodique. Au mieux, c'est une phase de l'opération traduisante à laquelle il pourra purement et simplement se soustraire. Toutefois, tant qu'il subsiste une opacité entravant la compréhension du texte, une recherche documentaire, même ponctuelle, reste indispensable.

Ensuite, il convient de cerner ce qu'on a besoin de savoir pour comprendre le texte à traduire. Dans l'exemple du train à lévitation magnétique, l'idéal serait que le traducteur possède les connaissances suivantes:

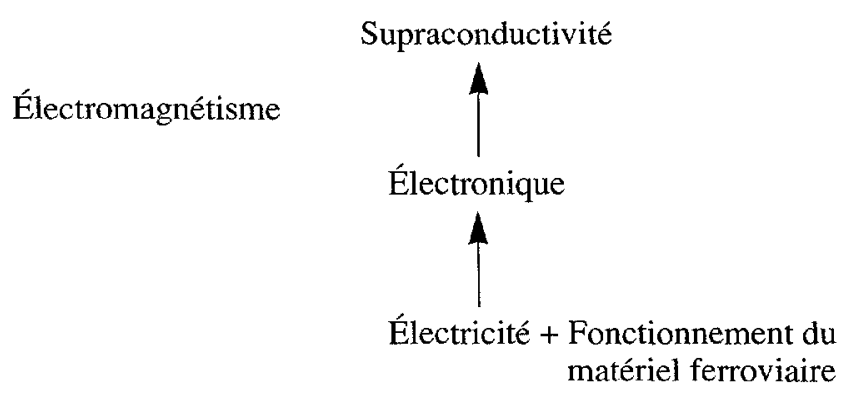

Il faut toutefois prendre conscience, d'une part, du temps nécessaire pour collecter, lire et assimiler tout ce savoir et, d'autre part, des impératifs de délais imposés au traducteur. Par ailleurs, le traducteur n'a pas besoin d'acquérir une compétence de conception ou d'exécution, celle que l'on exige par exemple d'un ingénieur, mais seulement une compétence de compréhension qui est beaucoup plus rapidement accessible.

Dans la réalité, le traducteur n'aborde jamais un texte sans avoir la moindre connaissance préalable concernant directement ou indirectement le sujet. Le traducteur technique, même non spécialisé, en raison même de son niveau de formation, possède normalement les notions de base de courant électrique, résistance, conducteur par opposition à isolant, etc.; il a déjà vu un train, a déjà manipulé un aimant, que ce soit pour aider sa grand-mère à ramasser des épingles ou pour placarder un mémo sur un tableau blanc; et par analogie avec l'hydroglisseur propulsé sur coussin d'air, il peut imaginer un train circulant sans contact ni frottement sur un rail ou entre deux glissières.

En outre, le texte lui-même apporte des informations qu'il comprend en les rattachant à des choses sues, qu'il s'agisse d'un savoir livresque ou expérimental. Si le traducteur est expérimenté dans une discipline connexe ou s'il a acquis les bases de la discipline concernée, sa recherche documentaire sera plus aisée et plus rapide, d'une part parce qu'il saura où chercher les informations dont il a besoin sans perdre de temps et, d'autre part, parce qu'il sera mieux à même d'appréhender des connaissances nouvelles pour lui dès lors qu'il pourra les rattacher à un savoir préalablement acquis. Toutefois, des connaissances connexes ou élémentaires ne doivent pas faire illusion et dissuader le traducteur de se lancer dans une recherche documentaire méthodique. Même si le traducteur a, par exemple, acquis une petite expérience en traduisant un texte sur les jonctions Josephson, et de ce fait sait ce qu'est la supraconductivité, il devra rechercher des explications sur l'électromagnétisme et le diamagnétisme pour pouvoir aborder efficacement la traduction du texte sur les trains à lévitation magnétique. 
Le traducteur doit éviter de se perdre dans de volumineux manuels et traités. Il n'a pas l'obligation de se constituer en totalité la pyramide des connaissances indiquées cidessus.

Contrairement à ce que Claude Bédard pense, (La traduction technique : principes et pratique, 1986), le traducteur technique n'a pas besoin de suivre un cours formel dans toute une série de disciplines. En effet, pour traduire un texte donné, il suffit au traducteur d'acquérir dans chaque discipline les éléments de connaissance de nature à lui permettre de construire sa propre pyramide, juste pour répondre à un besoin ponctuel. Là encore, ce que le traducteur doit acquérir n'est pas une somme absolue mais une valeur relative, fonction des connaissances qu'il possède déjà, des informations apportées par le texte luimême et des connaissances que l'auteur du texte original présupposait chez ses lecteurs. En tant que relais dans la chaîne de communication, le traducteur doit, en tout état de cause, posséder les connaissances présupposées par l'auteur chez ses lecteurs, faute de quoi il ne pourrait comprendre le message ou l'information contenue dans le texte à traduire.

Enfin, le traducteur doit mettre en auvre une démarche méthodique. Celle-ci suit deux grands axes: des généralités aux détails et de la vulgarisation à l'information spécialisée.

Reprenons l'exemple des trains à lévitation magnétique. Inutile de se lancer dans l'étude du phénomène de supraconductivité si l'on a oublié comment se fait le passage du courant électrique et ce qui fait qu'un matériau est conducteur ou au contraire isolant. Il existe bien un ordre logique à respecter: d'abord les notions d'atomistique expliquant la conduction électrique, puis le comportement des électrons à une jonction, avant d'aborder la supraconductivité.

Les sources documentaires sont multiples et l'aide qu'elles fournissent est très diverse. Concrètement, on commence par s'assurer une base solide en se reportant soit à un petit ouvrage de synthèse de type Que Sais-je faisant le point sur la conduction électrique, soit à un manuel de physique de classe de seconde en se limitant à l'exposé du cours sur la conduction électrique, sans s'attarder sur les formules, théorèmes et problèmes, soit encore à une encyclopédie.

L'encyclopédie est un outil très précieux qui se prête à une consultation libre, au gré du lecteur, qui peut naviguer au sein de cette fantastique somme d'informations selon ses besoins. Pour notre exemple, on débute par l'article le plus générique «Conduction de l'électricité». On lit attentivement le chapitre sur la conduction dans les solides, puis on parcourt rapidement le paragraphe sur la propriété des métaux, et on s'arrête là. En effet, les chapitres sur la conduction dans les liquides et la conduction dans les gaz n'apportent pas d'informations pertinentes supplémentaires.

On poursuit la recherche par une étude des semiconducteurs. Si l'on a choisi de consulter l'Encyclopaedia Universalis, la liaison avec l'article précédent est assurée dans l'introduction de l'article intitulé «Semiconducteurs», qui est une mine d'informations pertinentes avec un premier chapitre sur la structure électronique des cristaux parfaits et des semiconducteurs, un deuxième sur les propriétés physiques et notamment la conductivité électrique et le troisième sur les dispositifs à semiconducteurs. Là, on peut s'arrêter à la fin du premier paragraphe consacré aux jonctions. On se sent alors armé pour entrer dans le vif du sujet et aborder l'article intitulé «Supraconductivité».

Toutefois, on s'aperçoit vite que si l'on a acquis les rudiments d'électronique indispensables, on bute sur une insuffisance de connaissances. À cette étape de la recherche documentaire, on peut combler rapidement ses lacunes en se reportant à l'excellent ouvrage de vulgarisation de Sven Ortoli et Jean-Pierre Pharabod, Le Cantique des quantiques 
(Éditions de la Découverte, 1984) par exemple. On se rend compte aussi que l'on manque de connaissances en électromagnétisme; des notions font obstacle à la compréhension et le contexte dans lequel on les rencontre indique que ce sont des concepts clés à élucider : diamagnétisme, effet Meissner, paires de Cooper. À ce stade, l'encyclopédie révèle ses limites.

Certes, l'encyclopédie ne peut répondre à toutes les interrogations du traducteur, mais c'est un outil formidable, d'une part, du fait de l'énorme somme de connaissances qu'elle renferme de façon très ordonnée et structurée et, d'autre part, du fait de la liberté totale dont jouit celui qui la consulte. En effet, on peut passer d'un article à l'autre et affiner sa recherche, à son gré, sans schéma prédéterminé imposé, pour répondre à un besoin ponctuel et singulier. C'est un fait que la consultation d'une encyclopédie peut donner lieu à une démarche exactement adaptée aux besoins du traducteur à un moment donné, face à un texte donné. En revanche, la consultation d'un manuel ou d'un traité, même muni d'un index, offre beaucoup moins de souplesse, car l'auteur impose alors son propre schéma de progression dans l'apport d'informations. Néanmoins, les manuels spécialisés sont d'un grand secours, notamment lorsque le traducteur a épuisé les ressources de l'encyclopédie pour son problème particulier de documentation. C'est le cas ici, où la consultation d'un traité d'électromagnétisme s'impose. Il ne s'agit pas de le lire de la première à la dernière page, mais plutôt de parcourir rapidement certains chapitres et d'étudier plus attentivement certains passages.

Enfin, le sujet étant très nouveau, on peut rechercher des articles récents publiés dans des revues techniques. Compte tenu des délais très longs de publication des ouvrages en librairie et de renouvellement des encyclopédies, les revues sont beaucoup plus à même de fournir des informations à jour sur des techniques de pointe.

Dans le domaine de la supraconductivité, on a le choix entre au moins 38 articles en français parus sur le sujet entre 1986 et 1989 dans des supports aussi divers que Sciences et Avenir, La Recherche, l'Usine Nouvelle, le Bulletin de la Société française de physique, et 35 articles en anglais parus au cours de la même période aussi bien dans des revues spécialisées comme ChemTech, Physics World, Physics Today, Physical Review, Europhysics Letters que dans des revues techniques générales telles que Scientific American, Nature, New Scientist, Science ou même dans la presse quotidienne comme le Financial Times.

Dans la grande majorité des cas, les sources documentaires écrites permettent de résoudre les problèmes de recherche d'informations. Il subsiste néanmoins des cas où le traducteur ne peut trouver la réponse à ses interrogations, généralement parce que le sujet relève d'une technique trop récente. La documentation orale prend alors le relais et le traducteur ne doit pas hésiter à interroger des spécialistes. Toutefois, ces derniers n'apprécient guère d'être dérangés par des néophytes et jugent leur temps précieux. C'est pourquoi, s'il veut obtenir des spécialistes des réponses utiles, le traducteur a intérêt à bien circonscrire et préparer ses questions en précisant à partir de quel point il ne comprend plus le fonctionnement d'un mécanisme ou le déroulement d'un processus, et ce qu'il a besoin exactement de savoir et de comprendre.

On remarque que, menée dans la langue de départ ou dans une langue tierce, la recherche documentaire joue un rôle clé dans la phase sémasiologique qui doit aboutir à l'appréhension du sens du texte. Menée parallèlement dans la langue de départ et dans la langue d'arrivée, cette démarche étaye non seulement la phase sémasiologique, mais aussi la phase onomasiologique, puisqu'elle fournit en même temps que des éléments de compréhension la terminologie et la phraséologie pertinentes. Ainsi donc, à condition bien sûr de consulter dans chaque langue des documents fiables, écrits par des spécialistes dans leur langue maternelle, la recherche documentaire permet de réduire considérablement, 
voire d'éviter la recherche terminologique ponctuelle. Cette dernière devient inutile puisque les problèrnes qu'elle est censée résoudre ont déjà trouvé leur solution dans la recherche documentaire menée dans les deux langues (de départ et d'arrivée).

La méthode interprétative de la traduction est souvent accusée de faire perdre du temps. C'est long de se documenter: il faut trouver les sources documentaires, les consulter et en assimiler le contenu. Il paraît beaucoup plus rapide de consulter un dictionnaire bilingue ou une banque de données, mais ensuite il faut du temps pour vérifier la validité des correspondances trouvées pour le contexte en question, sans parler du temps nécessaire pour assembler toutes les correspondances et reconstruire un sens. En fin de compte, la méthode interprétative fait gagner du temps. Au lieu de procéder en deux temps d'abord faire de la recherche terminologique puis essayer de comprendre - il est infiniment plus rapide et efficace de privilégier la compréhension du texte par le biais de l'étude documentaire. En effet, cette démarche foumit d'un même coup les résultats des deux phases de travail: comprendre puis réexprimer. Bien entendu, la méthode interprétative qui, appliquée à la traduction technique, appelle souvent une recherche documentaire, ne permet de gagner du temps que si l'on sait s'arrêter dans la recherche à l'issue de ce qui est nécessaire et au niveau de ce qui est suffisant.

\section{CONDITIONS SUFFISANTES}

La question qui revient constamment est de savoir quand arrêter la recherche documentaire. L'ignorance du traducteur (et sans doute de tout homme) est telle et l'ampleur du domaine de la technique est également telle, qu'on peut imaginer un individu passant sa vie à se documenter sans jamais se décider à traduire une ligne !

La première réponse consiste à dire qu'on peut arrêter la recherche documentaire lorsqu'on a compris le texte à traduire. Mais il convient alors de déterminer le seuil de compréhension à partir duquel la traduction devient possible. C'est sans doute le terme compréhension qui est ici le plus difficile à définir. Si l'on se réfère au Dictionnaire de didactique des langues de R. Galisson et D. Coste, on lit:

Compréhension n. f. : S'agissant de communication linguistique : opération mentale, résultat du décodage d'un message, qui permet à un lecteur (compréhension écrite) ou à un auditeur (compréhension orale) de saisir la signification que recouvrent les signifiants écrits ou sonores.

Cette définition ne correspond qu'à une première étape de la compréhension, qui est la compréhension des concepts. Cette étape est certes nécessaire, mais elle n'est nullement suffisante pour traduire, car la traduction porte sur tout un discours et non sur des concepts juxtaposés. Il est donc indispensable, pour pouvoir traduire, de comprendre non seulement les concepts qui, eux, ont une portée générale, mais aussi les relations entre les concepts qui sont singulières, propres au discours hic et nunc.

C'est pourquoi, dans la recherche documentaire, nous privilégions la consultation d'encyclopédies qui expliquent des phénomènes, des processus, des mécanismes et, en quelque sorte, les mettent en scène, c'est-à-dire les décrivent dans leur contexte naturel et réel, et facilitent l'établissement de relations plus larges entre eux par le biais des corrélats. En revanche, les dictionnaires thématiques unilingues donnent des définitions de concepts isolés, hors contexte. On peut dire qu'ils fournissent des éléments de compréhension au sens où l'entendent R. Galisson et D. Coste. Ce sont des aides utiles ponctuellement, mais qui ne permettent pas d'induire une compréhension suffisante pour traduire.

La compréhension, telle qu'elle est définie par Jean Dubois et ses collaborateurs dans son Dictionnaire de linguistique, se rapproche davantage de ce dont le traducteur a besoin : 
Compréhension : On dit qu'un énoncé est compris quand la réponse de l'interlocuteur dans la communication instaurée par le locuteur est conforme à ce que ce dernier en attend, que la réponse faite soit un énoncé ou un acte.

Le traducteur, maillon intermédiaire dans la chaîne de communication entre l'auteur et le lecteur, est le premier interlocuteur de l'auteur, et sa propre réponse, concrétisée par l'expression de l'énoncé dans une autre langue, doit être de nature à susciter chez le lecteur final une réponse conforme à l'attente de l'auteur. La traduction d'un mode d'emploi ou d'un manuel d'instructions concernant l'installation, le transport ou la maintenance d'un matériel en est un exemple type.

Mais dans ce cas, on ne peut constater qu'a posteriori qu'il y a eu compréhension de la part du traducteur, par exemple si l'utilisateur réussit à faire marcher la machine. En règle générale, le traducteur n'a pas la possibilité d'expérimenter lui-même l'exécution des modes d'emploi pour vérifier s'il a bien compris le texte. Par ailleurs, dans la vie professionnelle, beaucoup de textes à traduire ne se prêtent pas à une expérience aussi concrète. Il faut donc trouver un moyen de s'assurer que la compréhension qu'on a du sujet est suffisante pour effectuer la traduction, et ce, dans des limites de temps compatibles avec les délais imposés par le donneur d'ouvrage.

On peut tester ses propres connaissances sur un thème en s'interrogeant systématiquement. La démarche de l'interrogation socratique, fondement de la maïeutique, visait à mettre en lumière un savoir infus dans l'esprit humain. En réalité, soumis à l'examen, ce prétendu savoir préalable se réduit à savoir ce qu'on ne sait pas. C'est en cela que la méthode de l'interrogation est intéressante ici. Par exemple, la question pourquoi ou comment posée en cascade permet de mettre en évidence non pas tant les éléments devenus transparents que les éléments qui restent opaques pour le traducteur, ce qui donne à ce dernier une orientation pour poursuivre son étude documentaire.

Cette démarche intellectuelle évoque les poupées russes : la réponse à chaque question amenant à entrer davantage dans le détail du développement d'un phénomène, du déroulement d'un processus, du fonctionnement d'un mécanisme, de la progression d'une argumentation, etc. Il faut poursuivre cette interrogation en règle jusqu'à ce que tous les éléments d'information explicites et présupposés dans le texte à traduire soient bien intégrés dans l'esprit du traducteur.

Cette idée d'intégration des concepts et des relations entre eux en un tout structuré dans l'esprit du traducteur conduit à énoncer un autre critère de suffisance de l'effort de recherche documentaire: on doit obtenir un système autonome. Dès lors qu'on dispose d'un système autonome, c'est-à-dire qu'on a acquis les connaissances nécessaires pour reconstruire un ensemble d'informations structuré dont les éléments sont interdépendants et forment un tout organisé, on peut considérer que l'on est parvenu à un stade de compréhension suffisant pour traduire.

Pour vérifier que l'on a réussi à se composer un système autonome, on peut projeter concrètement l'image mentale que l'on a du contenu du texte sous forme de schéma, de dessin ou de discours. Est-on en mesure de raconter à quelqu'un ce qu'il y a dans le texte à traduire? Dans l'affirmative, c'est que le texte est compris; dans la négative, il convient de clarifier les zones d'ombre en reprenant et en complétant l'étude documentaire.

Reprenons l'exemple du train à lévitation magnétique. Une recherche documentaire a été effectuée et elle est satisfaisante, ou bien aucune recherche documentaire n'a été effectuée et elle aurait été effectivement superflue, si l'on est capable de dessiner, même maladroitement, le train avec la position des bobines et des aimants - ceux qui servent à la lévitation et ceux qui servent à la propulsion - et à figurer schématiquement son fonctionnement. Si tous les éléments d'information se mettent en place comme les pièces 
d'un puzzle, alors le traducteur a compris le texte et il peut en exécuter la traduction. Tant que subsistent des incertitudes, qu'on hésite dans le dessin ou le schéma, qu'on ne parvient pas à organiser logiquement les informations qu'on relate à un interlocuteur fictif ou qu'on ne sait répondre aux questions d'un interlocuteur réel à propos du texte, on a la preuve concrète qu'on n'a pas obtenu un système autonome. Les conditions suffisantes de la recherche documentaire ne sont alors pas remplies, et il faut poursuivre l'effort.

Ce sont là des critères qui ont le mérite d'être concrets et tangibles. Il est bien entendu impossible d'énoncer des critères quantitatifs pour déterminer l'ampleur nécessaire et suffisante de la recherche documentaire, puisque la compréhension est un mode de connaissance d'ordre intuitif et synthétique. De fait, la compréhension implique bien un processus d'intégration d'éléments de savoir. Finalité et aboutissement de la recherche documentaire, la compréhension sert en fait deux objectifs: l'un, ponctuel, qui est de pouvoir effectuer la traduction d'un texte, et l'autre, plus général, qui est de se constituer un bagage de connaissances qu'il sera possible de mobiliser pour contribuer à la compréhension d'autres textes, ultérieurement. C'est bien la compréhension qui est la clé de voûte de tout l'édifice, c'est elle qui permet d'établir des relations et une cohésion entre les différents éléments de savoir que l'on acquiert jour après jour. 